ESAIM: PROCEEDINGS, December 2008, Vol. 25, p. 19-28

E. Cancès, S. Faure, B. Graille, Editors

\title{
EXACT CONTROLLABILITY AND OUTPUT FEEDBACK STABILIZATION OF A BIMORPH MIRROR
}

\author{
Pierre Le Gall ${ }^{1}$, Christophe Prieur ${ }^{2}$ and Lionel Rosier ${ }^{1}$
}

\begin{abstract}
We consider a large bimorph mirror which is composed of three layers: a purely elastic layer, a layer equipped with a distribution of sensor piezoelectric inclusions, and a layer equipped with a distribution of actuator piezoelectric inclusions. Such a device is modelized by a system of two coupled PDE, the first one involving a second-order operator without time derivative, and the second one being a plate equation. The controllability properties are investigated for both the $1 \mathrm{D}$ model and the 2D model, and an output feedback law is proposed for the stabilization of the 1D model.

Résumé. Nous considérons un miroir bimorphe qui est composé de trois couches: une couche purement élastique, une couche munie d'une distribution de capteurs piézoélectriques, et une autre munie d'une distribution d'actionneurs piézoélectriques. Un tel équipement est modélisé par un système de deux EDP couplées, la première étant écrite à l'aide d'un opérateur du second ordre sans dérivation en temps, et la deuxième étant l'équation des plaques. Nous étudions la contrôlabilité des modèles $1 \mathrm{D}$ et $2 \mathrm{D}$, et nous proposons un retour de sortie stabilisant le modèle $1 \mathrm{D}$.
\end{abstract}

\section{INTRODUCTION}

The general problem under study in this paper is the control and the stabilization of a bimorph mirror. Such a structure is an active multi-layered flexible plate (see Figure 1):

- a layer is assumed to be a purely flexible plate (this is the mirror);

- another layer is equipped with a distribution of piezoelectric inclusions, which are used as actuators;

- the last layer is also equipped with a distribution of piezoelectric inclusions, which are used as sensors.

. The thickness of the plate is neglected.

Such a device is used in Adaptive Optics with large ground-based telescopes. Recall that the main goal of Adaptive Optics is to compensate in real time for random wavefront disturbances [3].

This kind of flexible structure has been investigated in [10]. A PDE-type model has been obtained by letting the characteristic dimension of the heterogeneities tend to zero in elastic plates including small inclusions. Twoscale convergence for homogenization as in [1] was used. The goal of the present paper is to investigate the controllability and the stabilization properties of that model.

There exists a wide literature for the controllability and the stabilization of flexible plates equipped with piezoelectric inclusions. See e.g. [6,15] where controllability results for the Bernoulli Euler equation are obtained by using the Hilbert Uniqueness Method and Diophantine approximations. Stabilization results for the beam equation are given in [2], [12] and [8].

1 Institut Elie Cartan, Université Henri Poincaré Nancy 1, B.P. 239, 54506 Vandœuvre-lès-Nancy Cedex, France

${ }^{2}$ LAAS-CNRS, Université de Toulouse, 7 avenue du colonel Roche, 31077 Toulouse, France

(C) EDP Sciences, SMAI 2008 
An important feature of the model considered here is that there are two coupled PDE of very different nature: the first one is defined by a second-order operator without any time derivative, and the second one is a Euler-Bernoulli equation.

The model under consideration will be investigated in $1 \mathrm{D}$ and in 2D.

1D MODEL (BEAM)

$$
\left\{\begin{array}{l}
-\partial_{x}^{2} u=-s \partial_{x} \varphi+a \partial_{x}^{2} \varphi \\
\partial_{t}^{2} w+\partial_{x}^{4} w+w=\partial_{x}^{2} \varphi \\
w(0, t)=w(L, t)=\partial_{x} w(0, t)=\partial_{x} w(L, t)=0, \\
u(0, t)=u(L, t)=0 .
\end{array}\right.
$$

In above system, $x \in(0, L)$ is the spatial coordinate and $t$ is time, $w$ stands for the transverse deflection of the beam, $u$ is the scalar longitudinal displacement, and $\varphi$ is the voltage applied to the inclusions of the actuator layer. The coefficients $a$ and $s$ denote some physical constants depending on the piezoelectric inclusions (see e.g. [10]). We shall assume that $a>0$ and that $s$ is any real number.

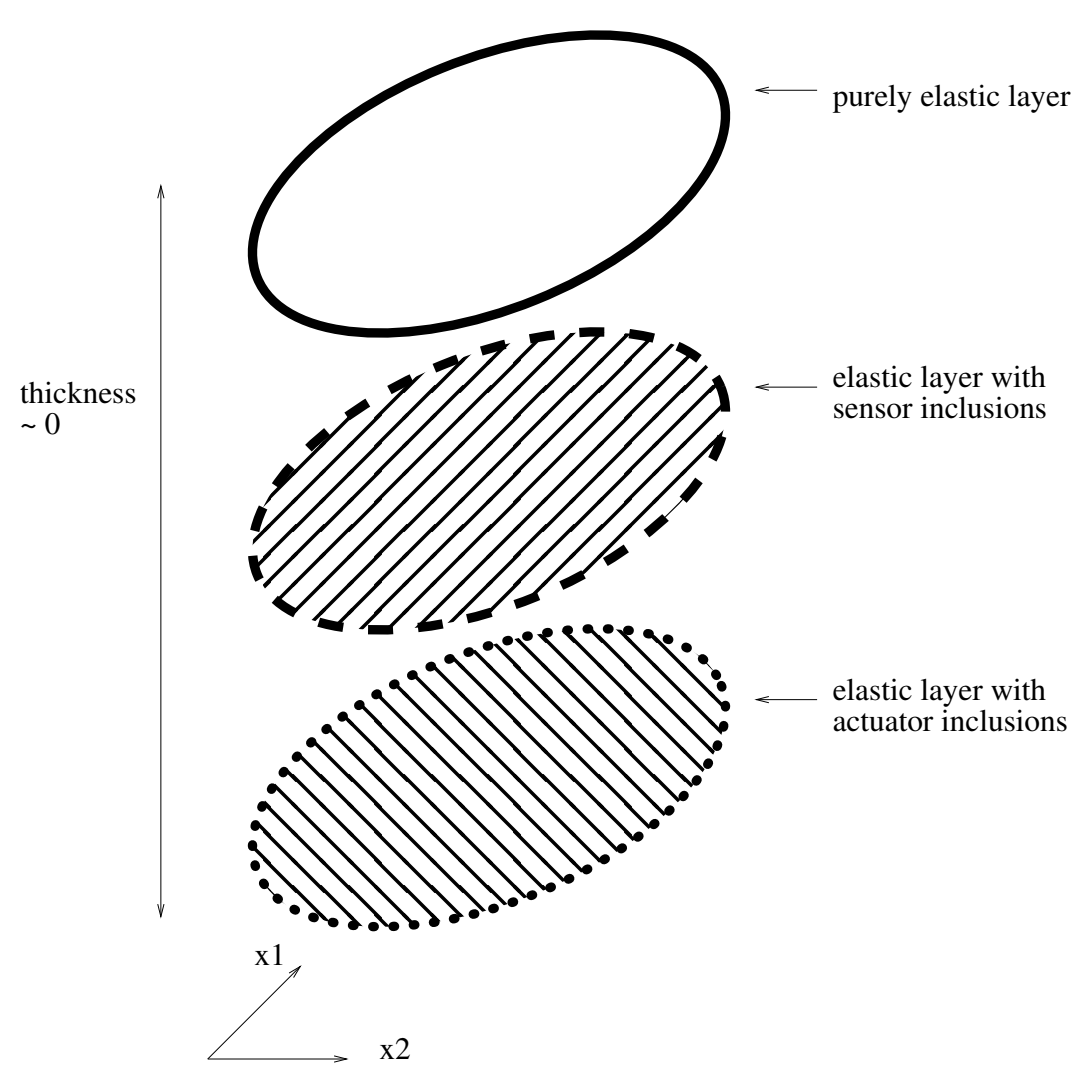

FIGURE 1. An active bimorph mirror 
2D MODEL (PLATE)

$$
\begin{aligned}
& -(\lambda+\mu) \partial_{1} \operatorname{div} U-\mu \Delta u_{1}=-s_{\beta} \partial_{\beta} \varphi+a_{\gamma \delta} \partial_{\gamma \delta}^{2} \varphi, \\
& -(\lambda+\mu) \partial_{2} \operatorname{div} U-\mu \Delta u_{2}=-t_{\beta} \partial_{\beta} \varphi+b_{\gamma \delta} \partial_{\gamma \delta}^{2} \varphi, \\
& \partial_{t}^{2} w+\Delta^{2} w+w=g_{\alpha \beta} \partial_{\alpha \beta}^{2} \varphi, \\
& w=\frac{\partial w}{\partial n}=0 \quad \text { on } \partial \Omega, \\
& u_{1}=u_{2}=0 \quad \text { on } \partial \Omega .
\end{aligned}
$$

In the above system, the spatial coordinates are denoted by $x=\left(x_{1}, x_{2}\right) \in \Omega$, where $\Omega \subset \mathbb{R}^{2}$ is a Lipschitzian bounded open set, $\partial_{1} \varphi=\partial \varphi / \partial x_{1}, \partial_{2} \varphi=\partial \varphi / \partial x_{2}, U=\left(u_{1}, u_{2}\right)$ stands for the 2D-longitudinal displacement, $\operatorname{div} U=\partial_{1} u_{1}+\partial_{2} u_{2}$, and Einstein's convention of summation for repeated indices has been adopted. As usual, $\lambda>0$ and $\mu>0$ stand for the Lame coefficients. We shall assume that the $2 \times 2$ symmetric matrices $A=\left(a_{\gamma \delta}\right)$, $B=\left(b_{\gamma \delta}\right)$, and $G=\left(g_{\alpha \beta}\right)$ are positive definite.

The paper is organized as follows. In Section 2, we prove the controllability of our model by treating separately the 1D model and the 2D model. In Section 3 we investigate the output feedback stabilization of the 1D model, the output being the distributed current field. Certain results of this paper have been announced in [9].

\section{Controllability properties}

\subsection{Toy problem}

We first investigate the possibility of achieving the exact controllability in finite time. The main feature of the control problem under study is the fact that we have to control the solution $u$ (resp. $U=\left(u_{1}, u_{2}\right)$ ) of an elliptic equation, in addition to the solution $w$ of the beam (resp. plate) equation. In a certain sense, $u$ or $U$ may be viewed as an "output" depending only on the input $\varphi$. The consideration of the following "toy problem" is quite illuminating. Consider three matrices $A \in \mathbb{R}^{n \times n}, B \in \mathbb{R}^{n \times m}$ and $C \in \mathbb{R}^{k \times m}$ and the following controlled linear system in finite dimension:

$$
\left\{\begin{aligned}
\dot{x} & =A x+B u \\
y & =C u
\end{aligned}\right.
$$

We shall say that the state $(x, y)$ is controllable in time $T$ if for all pairs $\left(x^{0}, y^{0}\right),\left(x^{T}, y^{T}\right)$ in $\mathbb{R}^{n} \times \mathbb{R}^{k}$ one may find a control input $u \in C\left([0, T] ; \mathbb{R}^{m}\right)$ and a solution $(x(t), y(t))$ of $(6)$ connecting $\left(x^{0}, y^{0}\right)$ to $\left(x^{T}, y^{T}\right)$. We shall say that the pair of matrices $(A, B)$ is controllable if $\operatorname{rank}\left[B, A B, A^{2} B, \ldots, A^{n-1} B\right]=n$ (see e.g. [14], [5]). Finally we shall say that the matrix $C$ is onto if the map $u \in \mathbb{R}^{m} \mapsto C u \in \mathbb{R}^{k}$ is onto. Then the following result holds.

Proposition 2.1. $(x, y)$ is controllable in time $T$ for any $T>0$ if and only if the pair of matrices $(A, B)$ is controllable and the matrix $C$ is onto.

Proof. The sense $\Rightarrow$ is obvious. Conversely, assume that the pair $(A, B)$ is controllable and that the matrix $C$ is onto. Let two pairs $\left(x^{0}, y^{0}\right)$ and $\left(x^{T}, y^{T}\right)$ be given in $\mathbb{R}^{n} \times \mathbb{R}^{k}$. In a first step we construct a trajectory $(\bar{x}, \bar{y})$ associated with a control $\bar{u}$ such that $\bar{y}(0)=y^{0}$ and $\bar{y}(T)=y^{T}$. To do this we pick some vectors $u^{0}$ and $u^{T}$ such that $y^{0}=C u^{0}$ and $y^{T}=C u^{T}$, and we set $\bar{u}(t):=(1-t / T) u^{0}+(t / T) u^{T}$. Let $\bar{x}$ denote the solution of $\dot{\bar{x}}=A \bar{x}+B \bar{u}, \bar{x}(0)=0$, and let $\bar{y}(t)=C \bar{u}(t)$. Performing the change of variables $\hat{x}=x-\bar{x}, \hat{u}=u-\bar{u}$, and $\hat{y}=y-\bar{y}$, we therefore have to look for a control input $\hat{u}$ and a pair $(\hat{x}, \hat{y})$ satisfying the system

$$
\begin{cases}\dot{\hat{x}} & =A \hat{x}+B \hat{u} \\ \hat{y} & =C \hat{u}\end{cases}
$$

and the constraints $C \hat{u}(0)=C \hat{u}(T)=0, \hat{x}(0)=x_{0}-\bar{x}(0)$, and $\hat{x}(T)=x_{T}-\bar{x}(T)$. The pair $(A, B)$ being controllable, it is well known that we may find a control input $\hat{u} \in C_{0}^{\infty}(0, T)$ steering $\hat{x}$ from $x_{0}-\bar{x}(0)$ to 
$x_{T}-\bar{x}(T)$.

Roughly speaking, to design the control we first construct a "static" control allowing to connect $y^{0}$ to $y^{T}$, and next we add to it a dynamical correction allowing to connect $x^{0}$ to $x^{T}$. The point is that this correction may be chosen with the additional constraint that it vanishes at both extremities of $[0, T]$. We shall see in the next section that this method works as well for the control of the bimorph mirror.

\subsection{Controllability of the $1 \mathrm{D}$ system.}

Let us introduce the spaces $V=H^{2}(0, L) \cap H_{0}^{1}(0, L), \tilde{V}=H_{0}^{2}(0, L), H=L^{2}(0, L)$ and the operator $\mathcal{A}(w, v)=\left(v,-w^{(4)}-w\right)$ with domain $\mathcal{D}(\mathcal{A})=\left(H^{4}(0, L) \cap H_{0}^{2}(0, L)\right) \times H_{0}^{2}(0, L) \subset \tilde{V} \times H$. Then we have the following result.

Theorem 2.2. Each pair $\left(\left(w^{0}, w^{1}\right), u^{0}\right),\left(\left(w^{0, T}, w^{1, T}\right), u^{T}\right)$ of triplets of functions in $\mathcal{D}(\mathcal{A}) \times V$ may be connected by a trajectory associated with a control function $\varphi \in C\left([0, T] ; H^{2}(0, L)\right)$.

Proof: As the system is time-reversible, we may assume without loss of generality that $w^{0, T}=w^{1, T}=u^{T}=$ 0 . Pick any triplet $\left(\left(w^{0}, w^{1}\right), u^{0}\right)$ in $\mathcal{D}(\mathcal{A}) \times V$. As suggested by Proposition 2.1 , we control first the variable $u$, and next the variable $w$. As $a \neq 0$, there exists a unique solution $\varphi^{0} \in V$ to the elliptic problem

$$
\left\{\begin{array}{l}
a \partial_{x}^{2} \varphi^{0}-s \partial_{x} \varphi^{0}=-\partial_{x}^{2} u^{0} \\
\varphi^{0}(0)=\varphi^{0}(L)=0 .
\end{array}\right.
$$

Let us set $\bar{\varphi}(t)=(1-t / T) \varphi^{0}$ and let $(\bar{w}, \bar{u})$ denote the solution of the system

$$
\left\{\begin{array}{l}
-\partial_{x}^{2} \bar{u}=-s \partial_{x} \bar{\varphi}+a \partial_{x}^{2} \bar{\varphi} \\
\partial_{t}^{2} \bar{w}+\partial_{x}^{4} \bar{w}+\bar{w}=\partial_{x}^{2} \bar{\varphi} \\
\bar{w}(0, t)=\bar{w}(L, t)=\partial_{x} \bar{w}(0, t)=\partial_{x} \bar{w}(L, t)=0 \\
\bar{u}(0, t)=\bar{u}(L, t)=0 \\
\bar{w}(x, 0)=\partial_{t} \bar{w}(x, 0)=0
\end{array}\right.
$$

Notice that $\bar{u}(x, 0)=u^{0}(x)$ and $\bar{u}(x, T)=0$, for $\bar{\varphi}(0)=\varphi^{0}$ and $\bar{\varphi}(T)=0$. As $\partial_{x}^{2} \bar{\varphi} \in C^{1}([0, T] ; H)$, we infer from a classical result in semigroup theory (see e.g. [4]) that $\left(\bar{w}, \partial_{t} \bar{w}\right) \in C([0, T] ; \mathcal{D}(\mathcal{A})) \cap C^{1}([0, T], \tilde{V} \times H)$. Next, we perform a change of unknown functions. We set $\hat{w}=w-\bar{w}, \hat{u}=u-\bar{u}$, and $\hat{\varphi}=\varphi-\bar{\varphi}$. Then the pair $(\hat{w}, \hat{u})$ solves

$$
\left\{\begin{array}{l}
-\partial_{x}^{2} \hat{u}=-s \partial_{x} \hat{\varphi}+a \partial_{x}^{2} \hat{\varphi} \\
\partial_{t}^{2} \hat{w}+\partial_{x}^{4} \hat{w}+\hat{w}=\partial_{x}^{2} \hat{\varphi} \\
\hat{w}(0, t)=\hat{w}(L, t)=\partial_{x} \hat{w}(0, t)=\partial_{x} \hat{w}(L, t)=0 \\
\hat{u}(0, t)=\hat{u}(L, t)=0 \\
\hat{w}(x, 0)=w^{0}(x), \partial_{t} \hat{w}(x, 0)=w^{1}(x)
\end{array}\right.
$$

and $\hat{\varphi}$ has to be designed in such a way that $\hat{u}(., 0)=0$ and $\left(\hat{w}(., T), \partial_{t} \hat{w}(., T), \hat{u}(., T)\right)=\left(-\bar{w}(., T),-\partial_{t} \bar{w}(., T), 0\right)$. In particular, the condition $\hat{\varphi}(., 0)=\hat{\varphi}(., T)=0$ is required. A classical result [11] on the controllability of the plate equation gives the existence of some control input $\hat{\varphi}$, which is compactly supported in time, and such that the corresponding trajectory fulfills all the above conditions. Alternatively, using the fact that the control is 
applied on the whole domain, we may design an explicit control input by specifying some trajectory $\left(\hat{w}, \hat{w}_{t}\right)$ in the class $C([0, H] ; \mathcal{D}(\mathcal{A})) \cap C^{1}([0, T] ; \tilde{V} \times H)$ which fulfills the above conditions. Next, $\hat{\varphi}(t)$ may be defined for each $t \in[0, T]$ as the solution of the elliptic problem

$$
\left\{\begin{array}{l}
\partial_{x}^{2} \hat{\varphi}=\partial_{t}^{2} \hat{w}+\partial_{x}^{4} \hat{w}+\hat{w} \\
\hat{\varphi}(0, t)=\hat{\varphi}(L, t)=0 .
\end{array}\right.
$$

To control the dynamical correction as suggested by Proposition 2.1, we need the following result.

Proposition 2.3. Let $v^{0}, v^{1}, v^{2}$ be three functions in $H^{4}(0, L) \cap H_{0}^{2}(0, L), H_{0}^{2}(0, L)$ and $L^{2}(0, L)$, respectively. There there exists a function $v \in C\left([0, T] ; H^{4}(0, L)\right) \cap C^{1}\left([0, T] ; H_{0}^{2}(0, L)\right) \cap C^{2}\left([0, T] ; L^{2}(0, L)\right)$ fulfilling v(0) $=$ $v^{0}, v^{\prime}(0)=v^{1}, v^{\prime \prime}(0)=v^{2}$ and $v(T)=v^{\prime}(T)=v^{\prime \prime}(T)=0$.

Proof of Proposition 2.3. Let $\left(\psi_{k}\right)_{k \geq 1}$ denote an orthonormal basis of $L^{2}(0, L)$ constituted of eigenfunctions for the operator $A w=w^{(4)}$ with the boundary conditions $w(0)=w(L)=w^{\prime}(0)=w^{\prime}(L)=0$. The eigenvalue associated with the function $\psi_{k}$ is denoted by $\lambda_{k}$. Pick any function $h \in C^{4}\left(\mathbb{R}^{+}\right)$such that $h(0)=h^{(3)}(0)=1$, $h^{\prime}(0)=h^{\prime \prime}(0)=h^{(4)}(0)=0$, and any function $g \in C^{4}\left(\mathbb{R}^{+}\right)$such that $g(t)=1$ for $t \leq T / 4$ and $g(t)=0$ for $t \geq T / 2$. If the functions $v^{0}, v^{1}$ and $v^{2}$ are decomposed along the $\psi_{k}$ 's as

$$
v^{0}=\sum_{k \geq 1} a_{k} \psi_{k}, v^{1}=\sum_{k \geq 1} b_{k} \psi_{k}, v^{2}=\sum_{k \geq 1} c_{k} \psi_{k}
$$

then $\sum_{k \geq 1}\left(k^{8}\left|a_{k}\right|^{2}+k^{4}\left|b_{k}\right|^{2}+\left|c_{k}\right|^{2}\right)<\infty$. The function $v$ is then defined as

$$
v(x, t)=g(t) \sum_{k \geq 1}\left(a_{k} h\left(\sqrt{\lambda_{k}} t\right)+\frac{b_{k}}{\sqrt{\lambda_{k}}} h^{\prime \prime}\left(\sqrt{\lambda_{k}} t\right)+\frac{c_{k}}{\lambda_{k}} h^{\prime}\left(\sqrt{\lambda_{k}} t\right)\right) \psi_{k}(x) .
$$

As $\lambda_{k} \sim C k^{4}$ as $k \rightarrow \infty$, one readily obtains that $v \in C\left([0, T] ; H^{4}(0, L)\right) \cap C^{1}\left([0, T] ; H_{0}^{2}(0, L)\right) \cap C^{2}\left([0, T] ; L^{2}(0, L)\right)$. The properties $v(0)=v^{0}, v^{\prime}(0)=v^{1}, v^{\prime \prime}(0)=v^{2}$ and $v(T)=v^{\prime}(T)=v^{\prime \prime}(T)=0$ are obvious.

The proof of Theorem 2.2 is completed by applying Proposition 2.3 to $v^{0}=w^{0}, v^{1}=w^{1}$, and $v^{2}=\partial_{x}^{4} \bar{w}(T)+\bar{w}(T)$ on the interval $[0, T / 2]$, and next to $v^{0}=-\bar{w}(T), v^{1}=\bar{w}_{t}(T)$ and $v^{2}=-\partial_{x}^{4} \bar{w}(T)-\bar{w}(T)$ on the interval $[T / 2, T]$ by reversing the time.

Remark 2.4. To simplify the exposition, we have imposed Dirichlet boundary conditions $(\varphi(0, t)=\varphi(L, t)=0)$ to the control input $\varphi$, but Neumann boundary conditions (i.e. $\partial_{x} \varphi(0, t)=\partial_{x} \varphi(L, t)=0$ together with e.g. $\left.\int_{0}^{L} \varphi(x, t) d x=0\right)$ may be taken instead.

\subsection{Controllability of the $2 \mathrm{D}$ system.}

Notice first that for any trajectory $(w(t), U(t))$ of $(1)-(5)$ associated with the control input $\varphi(t), t \in[0, T]$, the control $\varphi$ has to be at each instant $t$ a solution of the following system of elliptic PDE

$$
\begin{aligned}
& -s_{\beta} \partial_{\beta} \varphi+a_{\gamma \delta} \partial_{\gamma \delta}^{2} \varphi=f_{1}, \\
& -t_{\beta} \partial_{\beta} \varphi+b_{\gamma \delta} \partial_{\gamma \delta}^{2} \varphi=f_{2},
\end{aligned}
$$

where $f_{i}:=-(\lambda+\mu) \partial_{i} \operatorname{div} U-\mu \Delta u_{i}$ for $i=1,2$. Obviously, for $\varphi$ to exist the functions $f_{1}$ and $f_{2}$ have to satisfy a compatibility condition, namely

$$
\left(-t_{\beta} \partial_{\beta}+b_{\gamma \delta} \partial_{\gamma \delta}^{2}\right) f_{1}=\left(-s_{\beta} \partial_{\beta}+a_{\gamma \delta} \partial_{\gamma \delta}^{2}\right) f_{2} .
$$

Conversely, (9) proves to be a sufficient condition for the existence of a solution of (7)-(8) when $\Omega$ is a rectangle. Indeed, the following result holds. 
Proposition 2.5. Assume that $\Omega=\left(0, L_{1}\right) \times\left(0, L_{2}\right)$ with $L_{i}>0$ for $i=1,2$, and that $s_{1} t_{2}-s_{2} t_{1} \neq 0$. Let $f_{1}$ and $f_{2}$ be two functions in $L^{2}(\Omega)$ such that (9) holds true. Then there exists a function $\varphi \in H^{2}(\Omega)$ which solves (7)-(8).

Proof. Scaling in space if needed, we may assume that $L_{1}=L_{2}=2 \pi$. Let us note $P_{1}=-s_{\beta} \partial_{\beta}+a_{\gamma \delta} \partial_{\gamma \delta}$, $P_{2}=-t_{\beta} \partial_{\beta}+b_{\gamma \delta} \partial_{\gamma \delta}$, and $\left[f_{i}\right]=(2 \pi)^{-2} \int_{(0,2 \pi)^{2}} f_{i}(x) d x$ for $i=1,2$. Assume first that $\left[f_{1}\right]=\left[f_{2}\right]=0$. Using Fourier series, one easily sees that there exists a unique function $\varphi \in H_{p e r}^{2}\left((0,2 \pi)^{2}\right)$ such that $P_{1} \varphi=f_{1}$ and $[\varphi]=0$. Let us note $\varphi=P_{1}^{-1} f_{1}$. Since the operators $P_{2}$ and $P_{1}^{-1}$ act as Fourier multipliers on periodic functions with zero mean value, they commute. Using (9) and the fact that $\left[f_{2}\right]=0$, we obtain

$$
P_{2} \varphi=P_{2} P_{1}^{-1} f_{1}=P_{1}^{-1} P_{2} f_{1}=P_{1}^{-1} P_{1} f_{2}=f_{2} .
$$

Thus, $\varphi$ solves (7)-(8). To prove the result in the general case, it is sufficient to find $\psi$ such that $P_{i} \psi=\left[f_{i}\right]$ for $i=1,2$. Pick $\psi(x)=m_{1} x_{1}+m_{2} x_{2}$. Then $P_{1} \psi=-s_{1} m_{1}-s_{2} m_{2}$ and $P_{2} \psi=-t_{1} m_{1}-t_{2} m_{2}$, and the result follows at once if $s_{1} t_{2}-s_{2} t_{1} \neq 0$.

It is not clear, however, that the condition (9) is sufficient in general to guarantee the existence of a solution of (7)-(8). We shall adopt the following

Definition 2.6. A quadruplet $\left(w^{0}, w^{1}, u_{1}, u_{2}\right) \in\left(H^{4}(\Omega) \cap H_{0}^{2}(\Omega)\right) \times H_{0}^{2}(\Omega) \times\left(H^{2}(\Omega) \cap H_{0}^{1}(\Omega)\right)^{2}$ will be said to be compatible if the system (7)-(8) possesses a solution $\varphi \in H^{2}(\Omega)$, the functions $f_{1}, f_{2}$ being defined as $f_{i}:=-(\lambda+\mu) \partial_{i} \operatorname{div}\left(u_{1}, u_{2}\right)-\mu \Delta u_{i}$ for $i=1,2$.

Then the following result holds true.

Theorem 2.7. Each pair $\left(w^{0}, w^{1}, u_{1}, u_{2}\right),\left(w^{0, T}, w^{1, T}, u_{1}^{T}, u_{2}^{T}\right)$ of compatible quadruplets may be connected by a trajectory associated with a control function $\varphi \in C\left([0, T] ; H^{2}(\Omega)\right)$.

Proof. The proof follows the same pattern as for Theorem 2.2. Once again, we may assume that the terminal quadruplet is $(0,0,0,0)$.

Step 1: Control of the static equations.

As the quadruplet $\left(w^{0}, w^{1}, u_{1}, u_{2}\right)$ is assumed to be compatible, there exists a function $\varphi^{0} \in H^{2}(\Omega)$ solving (7)-(8). We set $\bar{\varphi}(t):=(1-t / T) \varphi^{0}$. Next, $\bar{w}$ is defined as the solution of the plate equation (3) (with $\bar{\varphi}$ substituted to $\varphi$ ) with the boundary conditions (4) and issuing from $(0,0)$, and $\bar{U}=\left(\bar{u}_{1}, \bar{u}_{2}\right)$ is the solution of the elliptic problem (1), (2) and (5).

Step 2: Control of the plate equation.

We perform the change of unknown functions $\hat{w}=w-\bar{w}, \hat{u}_{i}=u_{i}-\bar{u}_{i}(i=1,2)$, and $\hat{\varphi}=\varphi-\bar{\varphi}$. Then $\hat{u}_{1}, \hat{u}_{2}, \hat{w}$ and $\hat{\varphi}$ have to fulfill (1)-(5). The constraints at time 0 and $T$ are respectively $\left(\hat{w}(0), \hat{w}_{t}(0), \hat{u}_{1}(0), \hat{u}_{2}(0)\right)=$ $\left(w^{0}, w^{1}, 0,0\right)$ and $\left(\hat{w}(T), \hat{w}_{t}(T), \hat{u}_{1}(T), \hat{u}_{2}(T)\right)=\left(-\bar{w}(T),-\bar{w}_{t}(T), 0,0\right)$. To conclude, we apply the following result whose proof is virtually the same as for Proposition 2.3.

Proposition 2.8. Let $v^{0}, v^{1}, v^{2}$ be three functions in $H^{4}(\Omega) \cap H_{0}^{2}(\Omega), H_{0}^{2}(\Omega)$, and $L^{2}(\Omega)$, respectively. Then there exists a function $v \in C\left([0, T] ; H^{4}(\Omega)\right) \cap C^{1}\left([0, T] ; H_{0}^{2}(\Omega)\right) \cap C^{2}\left([0, T] ; L^{2}(\Omega)\right)$ fulfilling $v(0)=v^{0}, v^{\prime}(0)=$ $v^{1}, v^{\prime \prime}(0)=v^{2}$ and $v(T)=v^{\prime}(T)=v^{\prime \prime}(T)=0$.

The proof of Theorem 2.7 is complete. 


\section{OUtPut StabilizATION OF AN ADAPTIVE MiRRoR}

We consider the system

$$
\begin{aligned}
& -\partial_{x}^{2} u=-s \partial_{x} \varphi+a \partial_{x}^{2} \varphi \\
& \partial_{t}^{2} w+\partial_{x}^{4} w+w=\partial_{x}^{2} \varphi \\
& w(0, t)=w(L, t)=\partial_{x} w(0, t)=\partial_{x} w(L, t)=0 \\
& u(0, t)=u(L, t)=0 \\
& w(x, 0)=w^{0}(x), \partial_{t} w(x, 0)=w^{1}(x)
\end{aligned}
$$

and introduce the output

$$
I=\partial_{t}\left(\partial_{x}^{2} w+c \partial_{x} u\right) .
$$

$I$ stands for the distributed current field measured through the layer equipped with piezoelectric sensors.

We are interested in the output feedback stabilization of (10)-(14), the control $\varphi$ being expressed as a function of the output $I$. In the framework of Adaptive Optics, this corresponds to the problem of the stabilization at the rest position of a large mirror equipped with piezoelectric sensors and actuators. This problem should be seen as a first step towards the tracking problem, for which a control input is designed so that the mirror converges to a given trajectory.

Let us introduce the operator $K: H^{2}(0, L) \rightarrow H^{2}(0, L) \cap H_{0}^{1}(0, L)$ defined by $K \varphi=u$, where $u$ solves (10) and (13). Straightforward calculations give, for all $x \in[0, L]$,

$$
u(x)=-a(\varphi(x)-\varphi(0))+s \int_{0}^{x} \varphi(y) d y+\left\{a(\varphi(L)-\varphi(0))-s \int_{0}^{L} \varphi(y) d y\right\} x / L .
$$

For each $f \in L^{1}(0, L)$, we denote $[f]:=L^{-1} \int_{0}^{L} f(x) d x$. We are in a position to state the main result of this section.

Theorem 3.1. Pick any number $k>0$ and define $\varphi$ as the unique solution of the problem

$$
\begin{aligned}
& \partial_{x}^{2} \varphi=k \partial_{t} \partial_{x}^{2} w \quad \text { in }(0, L) \\
& \varphi(0)=\varphi(L), \quad[\varphi]=0
\end{aligned}
$$

so that $\varphi=k\left(\partial_{t} w-\left[\partial_{t} w\right]\right)$, and $\partial_{x}^{2} \varphi=k \partial_{t} \partial_{x}^{2} w$. Then for any $\left(w^{0}, w^{1}\right) \in\left(H^{4}(0, L) \cap H_{0}^{2}(0, L)\right) \times H_{0}^{2}(0, L)$, there exists a unique solution $(u, w)$ of (10)-(14) such that $u \in C\left(\mathbb{R}^{+} ; H^{2}(0, L) \cap H_{0}^{1}(0, L)\right), w \in C\left(\mathbb{R}^{+} ; H^{4}(0, L) \cap\right.$ $\left.H_{0}^{2}(0, L)\right) \cap C^{1}\left(\mathbb{R}^{+} ; H_{0}^{2}(0, L)\right)$. Moreover, there exist some constants $C>0, \mu>0$ such that for any $\left(w^{0}, w^{1}\right) \in$ $\left(H^{4}(0, L) \cap H_{0}^{2}(0, L)\right) \times H_{0}^{2}(0, L)$

$$
\|u(t)\|_{H^{2}(0, L)}+\|w(t)\|_{H^{4}(0, L)}+\left\|w_{t}(t)\right\|_{H^{2}(0, L)} \leq C\left(\left\|w^{0}\right\|_{H^{4}(0, L)}+\left\|w^{1}\right\|_{H^{2}(0, L)}\right) e^{-\mu t} \quad \forall t \geq 0 .
$$

Finally, $\varphi$ may be viewed as an output feedback, being the only solution of the system

$$
\begin{aligned}
& k^{-1} \partial_{x}^{2} \varphi+c \partial_{t} \partial_{x} K \varphi=I \\
& \varphi(0)=\varphi(L) . \quad[\varphi]=0 .
\end{aligned}
$$

Proof. Let us introduce the spaces $V=H_{0}^{2}(0, L), H=L^{2}(0, L)$, and the operator $\mathcal{A}(w, v)=\left(v,-w^{(4)}-w+k v^{\prime \prime}\right)$ with domain $\mathcal{D}(\mathcal{A})=\left(H^{4}(0, L) \cap H_{0}^{2}(0, L)\right) \times H_{0}^{2}(0, L) \subset V \times H$, the space $V \times H$ being endowed with the scalar product

$$
\langle(w, v),(\tilde{w}, \tilde{v})\rangle_{V \times H}=\int_{0}^{L}\left(w^{\prime \prime} \tilde{w}^{\prime \prime}+w \overline{\tilde{w}}\right) d x+\int_{0}^{L} v \overline{\tilde{v}} d x
$$


We have, for all $(w, v) \in \mathcal{D}(\mathcal{A})$,

$$
\langle\mathcal{A}(w, v),(w, v)\rangle_{V \times H}=-k \int_{0}^{L}\left|v^{\prime}\right|^{2} d x \leq 0,
$$

and thus $\mathcal{A}$ is dissipative. It is easily seen that $\mathcal{A}$ generates a continuous semigroup of contraction in $V \times H$. Let us prove the exponential stability in $V \times H$. According to Huang-Prüss theorem (see [7] and [13]), it is sufficient to check the two conditions

$$
\begin{aligned}
& i \mathbb{R} \subset \rho(\mathcal{A}) \\
& \sup _{\beta \in \mathbb{R}}\left\|(i \beta-\mathcal{A})^{-1}\right\|<\infty .
\end{aligned}
$$

First, the spectrum of $\mathcal{A}$ is constitued only of eigenvalues, since $\mathcal{A}^{-1}$ is compact. To prove (23) we argue by contradiction, assuming the existence of an eigenvalue of $\mathcal{A}$ of the form $i \beta$. Pick $(w, v) \in \mathcal{D}(\mathcal{A}) \backslash\{(0,0)\}$ such that $(i \beta-\mathcal{A})(w, v)=0$. Then

$$
0=\langle(i \beta-\mathcal{A})(w, v),(w, v)\rangle_{V \times H}=i \beta\left(\int_{0}^{L}\left(\left|w^{\prime \prime}\right|^{2}+|w|^{2}+|v|^{2}\right) d x\right)+k \int_{0}^{L}\left|v^{\prime}\right|^{2} d x
$$

hence $v=0$. (Recall that $v(0)=v(L)=0$.) On the other hand, $(i \beta-\mathcal{A})(w, v)=0$ yields $w^{(4)}+w=0$, which, combined to (12), gives $w=0$. The contradiction completes the proof of (23). Let us now proceed to the proof of (24). Argue again by contradiction. If (24) is false, then there exist $\beta_{n} \in \mathbb{R},\left(w_{n}, v_{n}\right) \in \mathcal{D}(\mathcal{A})$ for $n=1,2, \ldots$ such that

$$
\left\|\left(w_{n}, v_{n}\right)\right\|_{V \times H}=1, \quad\left|\beta_{n}\right| \rightarrow+\infty \text { as } n \rightarrow \infty
$$

and if we set $\left(f_{n}, g_{n}\right)=\left(i \beta_{n}-\mathcal{A}\right)\left(w_{n}, v_{n}\right)$,

$$
\left(f_{n}, g_{n}\right) \rightarrow(0,0) \text { in } V \times H
$$

i.e.

$$
\begin{aligned}
& i \beta_{n} w_{n}-v_{n}=f_{n} \rightarrow 0 \quad \text { in } V \\
& i \beta_{n} v_{n}+w_{n}^{(4)}+w_{n}-k v_{n}^{\prime \prime}=g_{n} \rightarrow 0 \quad \text { in } H .
\end{aligned}
$$

Using (22), (25) and (26), we obtain

$$
k \int_{0}^{L}\left|v_{n}^{\prime}\right|^{2} d x=\operatorname{Re}\left\langle\left(i \beta_{n}-\mathcal{A}\right)\left(w_{n}, v_{n}\right),\left(w_{n}, v_{n}\right)\right\rangle_{V \times H} \rightarrow 0 \quad \text { as } n \rightarrow \infty .
$$

On the other hand, (27) and (28) give, as $n \rightarrow \infty$,

$$
\begin{aligned}
& i \beta_{n}\left\|w_{n}\right\|_{V}^{2}-\left(v_{n}, w_{n}\right)_{V} \rightarrow 0, \\
& i \beta_{n}\left\|v_{n}\right\|_{H}^{2}+\left(w_{n}^{(4)}+w_{n}-k v_{n}^{\prime \prime}, v_{n}\right)_{H} \rightarrow 0 .
\end{aligned}
$$

Taking the difference of (30)-(31), we arrive to

$$
i \beta_{n}\left(\left\|w_{n}\right\|_{V}^{2}-\left\|v_{n}\right\|_{H}^{2}\right)-2 \operatorname{Re}\left(v_{n}, w_{n}\right)_{V}-k\left\|v_{n}^{\prime}\right\|_{H}^{2} \rightarrow 0 .
$$

Taking the imaginary part in (32) yields

$$
\beta_{n}\left(\left\|w_{n}\right\|_{V}^{2}-\left\|v_{n}\right\|_{H}^{2}\right) \rightarrow 0
$$


Therefore, using (25), we conclude that

$$
\lim _{n \rightarrow \infty}\left\|w_{n}\right\|_{V}^{2}=\lim _{n \rightarrow \infty}\left\|v_{n}\right\|_{H}^{2}=\frac{1}{2}
$$

which, combined to (29), leads to a contradiction. The proof of (24) is achieved. By Huang-Prüss theorem (see [7] and [13]), there exist some constants $C>0, \mu>0$ such that

$$
\left\|\left(w, w_{t}\right)(t)\right\|_{V \times H} \leq C\left\|\left(w^{0}, w^{1}\right)\right\|_{V \times H} e^{-\mu t} \quad \forall\left(w^{0}, w^{1}\right) \in V \times H .
$$

This yields

$$
\left\|\mathcal{A}\left(w, w_{t}\right)(t)\right\|_{V \times H} \leq C\left\|\mathcal{A}\left(w^{0}, w^{1}\right)\right\|_{V \times H} e^{-\mu t} \quad \forall\left(w^{0}, w^{1}\right) \in \mathcal{D}(\mathcal{A})
$$

and (19) follows, for $\|u\|_{H^{2}(0, L)} \leq C\|\varphi\|_{H^{2}(0, L)} \leq C\left\|w_{t}\right\|_{H^{2}(0, L)}$.

It follows from (15) and the definition of the operator $K$ that (17)-(18) is equivalent to (20)-(21).

Remark 3.2. When $c=0$, then (20)-(21) reduces to an elliptic problem. When $c \neq 0$, using (16), it is easily seen that

$$
\partial_{t} \partial_{x} K \varphi=-a \partial_{t} \partial_{x} \varphi+s \partial_{t} \varphi
$$

If in addition $s=0$, then $\varphi$ is the solution of the first order hyperbolic system

$$
\begin{aligned}
& k^{-1} \partial_{x} \varphi-a c \partial_{t} \varphi=\partial_{x}^{-1} I \\
& \varphi(0)=\varphi(L), \quad[\varphi]=0
\end{aligned}
$$

where $\partial_{x}^{-1} f:=\int_{0}^{x} f(y) d y-\left[\int_{0}^{x} f(y) d y\right]$.

\section{REFERENCES}

[1] G. Allaire. Homogenization and two-scale convergence. SIAM J. Math. Anal., 23(6):1482-1518, 1992.

[2] K. Ammari, A. Henrot, and M. Tucsnak. Asymptotic behaviour of the solutions and optimal location of the actuator for the pointwise stabilization of a string. Asymptot. Anal., 28(3-4):215-240, 2001.

[3] L. Baudouin, C. Prieur, F. Guignard, and D. Arzelier. Robust control of a bimorph mirror for adaptive optics system. J. of Applied Optics, to appear, 2008.

[4] T. Cazenave and A. Haraux. An introduction to semilinear evolution equations, volume 13 of Oxford Lecture Series in Mathematics and its Applications. The Clarendon Press Oxford University Press, New York, 1998. Translated from the 1990 French original by Yvan Martel and revised by the authors.

[5] J.-M. Coron. Control and nonlinearity, volume 136 of Mathematical Surveys and Monographs. American Mathematical Society, Providence, RI, 2007.

[6] E. Crépeau and C. Prieur. Control of a clamped-free beam by a piezoelectric actuator. ESAIM Control Optim. Calc. Var., 12:545-563, 2006.

[7] F.-L. Huang. Characteristic conditions for exponential stability of linear dynamical systems in Hilbert spaces. Ann. Differential Equations, 1(1):43-56, 1985.

[8] P. Le Gall, C. Prieur, and L. Rosier. Stabilization of a clamped-free beam with collocated piezoelectric sensor/actuator. Internat. J. Control, 80(8):1201-1216, 2007.

[9] Pierre Le Gall, Christophe Prieur, and Lionel Rosier. Stabilization of a clamped-free beam with collocated piezoelectric sensor/actuator. Int. J. Tomogr. Stat., 6(S07):104-109, 2007.

[10] M. Lenczner and C. Prieur. Asymptotic model of an active mirror. Int. J. Tomogr. Stat., 5(W07):68-72, 2007.

[11] J.-L. Lions. Contrôlabilité exacte, perturbations et stabilisation de systèmes distribués. Tome 1, volume 8 of Recherches en Mathématiques Appliquées [Research in Applied Mathematics]. Masson, Paris, 1988. Contrôlabilité exacte. [Exact controllability], With appendices by E. Zuazua, C. Bardos, G. Lebeau and J. Rauch.

[12] Z. Liu and S. Zheng. Semigroups associated with dissipative systems, volume 398 of Research Notes in Mathematics. Chapman \& Hall/CRC, 1999.

[13] J. Prüss. On the spectrum of $C_{0}$-semigroups. Trans. Amer. Math. Soc., 284(2):847-857, 1984.

[14] E. Sontag. Mathematical Control Theory: Deterministic Finite Dimensional Systems. Springer, New York, second edition, 1998. 
[15] M. Tucsnak. Regularity and exact controllability for a beam with piezoelectric actuator. SIAM J. Control Optim., 34(3):922930, 1996. 